\title{
Who do they think they are? Wnt-responsive cells reveal their family trees
}

\author{
Liliana Ordonez and Matthew J Smalley*
}

\begin{abstract}
The nature of stem and progenitor cells in the mammary epithelium, and the relevance of cleared fat pad transplantation as a functional assay for them, has been thrown into doubt by recent lineage-tracking studies. Now two new studies based on tracking the progeny of Wnt-responsive cells are starting to help make sense of this fascinating problem.
\end{abstract}

\section{Background}

The mammary gland undergoes dramatic morphological changes during puberty, pregnancy and involution. Transplantation studies of tissue fragments into a cleared mammary fat pad pioneered by DeOme and colleagues showed reconstitution of the mammary gland [1], demonstrating the presence of mammary stem cells. Work on serial transplantation of small numbers of purified basal mammary epithelial cells showed that they could regenerate the entire mammary epithelial tree with high efficiency and, indeed, that a single basal cell could do so [2-5].

These studies suggested the presence of multipotent mammary stem cells, located in the basal compartment. In contrast, purified luminal cells have limited fat pad reconstitution ability, although c-Kit-positive luminal cells were capable of forming small epithelial outgrowths in the transplant assay [5]. This model was recently challenged by Van Keymeulen and colleagues, who suggested that the basal and luminal lineages were uncoupled in the adult mammary epithelium and that each lineage contained a unipotent stem cell population [6]. The lineage contribution of stem/progenitor cells during pregnancy was unclear, however, as Van Keymeulen and colleagues were able to label very few luminal cells

*Correspondence: SmalleyMJ@Cardiff.ac.uk

European Cancer Stem Cell Research Institute and Cardiff School of Biosciences, Cardiff University, Museum Avenue, Cardiff CF10 3AX, UK for lineage tracking - many fewer than the number of pregnancy-responsive progenitors, thought to be luminal, in the gland $[5,6]$. These progenitors generate alveoli, containing milk-secreting alveolar luminal cells and contractile myoepithelial cells, during pregnancy. Previous studies on lineage tracking of pregnancy-responsive mammary epithelial cells suggested that luminal pregnancy-responsive progenitors could contribute to both luminal and myoepithelial layers in the alveoli [7].

Our understanding of the relationship of stem and progenitor populations in the mammary epithelium with the different epithelial lineages is therefore currently very much in flux; two new publications examining the lineage contributions of Wnt-responsive cells are starting to clear these muddy waters.

\section{The articles}

Van Amerongen and colleagues reported the coexistence of unipotent and multipotent stem cells in the mouse mammary gland [8], helping draw together apparently disparate points of view. The authors utilised the promoter of Axin2, a Wnt target gene, to drive expression of an inducible Cre recombinase. They performed lineagetracing analyses by generating Axin $2^{\mathrm{CreERT2/+}} ; \operatorname{Ros} 22^{\text {lacZ/+ }}$ and Axin $2^{\mathrm{CrERRT} 2 /+} ; \operatorname{Rosa} 26^{\mathrm{mTm} /+}$ mouse models, which enabled Wnt-responsive cells to be labelled by LacZ staining or fluorescence following induction of Cre activity. Labelled cells were tracked in situ after a single tamoxifen pulse to activate the Cre and were analysed at different developmental stages.

To analyse Wnt-responsive cell activity in the embryo, a single pulse was administered on embryonic day 12.5 , 14.5 or 17.5 and tissue was analysed once the animals reached adulthood. Labelled cell clones were restricted to the luminal cell lineage, implying the presence of unipotent stem cells. Interestingly, however, when a single pulse was administrated to pre-pubescent mice (postnatal days 14 to 16), which were then allowed to develop to adulthood before analysis, labelled clones were restricted to the basal cell lineage. This observation again supported the presence of unipotent stem cells, but also suggested a switch in Wnt-responsive cells after birth. 
Transplantation of these unipotent stem cells from prepubescent mice into cleared fat pads resulted in the complete regeneration of mammary glands. When Cre activity was induced during puberty, labelled cells were located in both the basal and luminal compartments of the terminal end buds, contributed to both the luminal and basal layers of the subtending ducts and underwent clonal expansion at pregnancy. However, the labelled clones in the luminal and basal layers were not adjacent and the authors interpreted this as indicating the presence of two unipotent Wnt-responsive stem cell populations, one luminal and one basal, during pubertal development. Induction of Cre activity in the adult gland resulted in labelling only of basal cells in the resting gland. However, if the animals underwent timed matings 1 week after induction, labelled cells could be observed both in basal and luminal layers. Moreover, these labelled cells were adjacent to each other, suggesting a clonal relationship. This suggested the presence of a pregnancyresponsive bipotent stem cell.

de Visser and colleagues also reported a switch in the Wnt-responsive target population in the mouse mammary gland [9]. In their mouse model (Lgr5-EGFP-IresCreERT2), the Wnt-responsive Lgr5 promoter drove expression of tamoxifen-inducible Cre as well as EGFP (to identify Cre-expressing cells). As with the model of Van Amerongen and colleagues, lineage tracing was achieved by Cre-dependent activation of fluorescent or LacZ reporter alleles. Cre activity was induced with a single pulse of tamoxifen either 1 or 12 days after birth (postnatal day 1 or 12) and analysis of the tissue was performed by flow cytometry 3 months post induction.

The results showed that when Cre activity was induced on postnatal day 1 , the labelled cells were luminal in the mature gland. Consistent with this observation, analysis of mammary glands from newborn mice carrying a LacZ transgene driven directly by the $\operatorname{Lgr} 5$ promoter (postnatal day 0 ) showed that LacZ expression was restricted to luminal cells. However, when Cre activity was induced at postnatal day 12 , the labelled cells were basal at 3 months old. Furthermore, in postnatal day 12 Lgr5-LacZ mice, the labelled population had also switched to a basal location. Finally, in mature adult mice the EGFP-positive (Wnt-responsive) population was also found to be basal, and in particular located in the CD49f $\mathrm{f}^{\mathrm{High}}$ basal stem cell population. EGFP-positive cells from adult mice were more highly enriched for cleared fat pad transplantation potential than EGFP-negative cells. The authors concluded that Wnt-responsive cells in the mouse mammary gland are not stem cells but rather are lineage-committed cells and that the results of the transplantation assay do not accurately reflect stem/progenitor behaviour in vivo. However, Cre activation and lineage tracking were not reported for the in situ mature adult gland.

\section{Are you a lineage tracker or a cell transplanter - or a mammary gland biologist?}

Taken together, these studies show that the target population for Wnt signalling in the embryonic or very early postpartum mammary gland is in the luminal cell lineage, whereas in older postpartum mice the target population switches and is in the basal lineage. A Wntresponsive population appears transiently in the luminal epithelium during pubertal development and then Wntresponsive cells are once again basally restricted in the adult. Importantly, both studies support the finding of Van Keymeulen and colleagues that the basal cell layer in the resting adult gland makes little contribution to the luminal cell layer, lending more weight to the view that both cell compartments, under normal physiological conditions, are maintained by separate stem/progenitor compartments. The term unipotent here is misleading, however, because the luminal cell layer contains a number of functional cell types and in a transplant situation the basal population (and to a lesser extent the luminal progenitor population) has the ability to generate all epithelial cell types in the gland. These cell populations therefore have multipotent potential but in the adult gland in situ this potential is normally restricted to either the basal or luminal lineages. Nevertheless, these findings must make us question the significance of cleared fat pad transplantation as an assay of stem cell potential.

Importantly, however, the findings of Van Amerongen and colleagues also provide evidence that there is indeed a physiological stem cell population which contributes to both basal and luminal layers in the alveoli during pregnancy. This hypothesis had been previously suggested by Boulanger and colleagues [7]. However, the timing of the labelling experiments of Van Amerongen and colleagues suggests that this pregnancy-responsive alveolar progenitor is a basal cell, whereas the work of Boulanger and colleagues suggested it was a luminal cell population. Indeed, much of the analysis of isolated mammary epithelial populations has supported the idea of a luminally located pregnancy-responsive progenitor. These discrepancies have yet to be resolved.

There are two caveats in the interpretation of all these studies. First, none of the lineage-tracing studies reported has labelled cap cells in terminal end buds and followed the fate of their progeny. Early morphological studies supported a model in which cap cells generated the subtending myoepithelial layer as well as the body cells, depending on the orientation of the plane of cell division [10-12]. The body cells then generated the luminal layer, implicating the cap cells as common stem cells for the whole mammary epithelium. Lineagetracing studies that specifically target this population are required to properly delineate the mammary hierarchy. 
Second, while these studies are fascinating, it is frustrating to try and place them in the context of previous work because the labelled populations being investigated are simply not well characterised. Some basic cell sorting of Cre-expressing and/or lineagemarked cells following by staining of cytospins and gene expression analysis would rapidly enable the unipotent stem cells/lineage-committed progenitors/pregnancyresponsive progenitors to be correlated with one of the epithelial subpopulations that flow cytometry has enabled us to isolated and characterise. Failure to achieve this correlation risks a rift in the community between the 'lineage trackers' and the 'cell transplanters'. If we can integrate our data properly, however, we have the potential for making the greatest advances seen for many years in our understanding of mammary gland biology.

Abbreviations

EGFP, enhanced green fluorescent protein.

\section{Competing interests}

The authors declare that they have no competing interests.

\section{Acknowledgments}

LO is funded by Biotechnology and Biological Sciences Research Council and is also partly supported by AstraZeneca. MJS is supported by Cardiff University, Breast Cancer Campaign and an EU Innovative Medicines Initiative consortium

Published: 10 December 2012

\section{References}

1. DeOme KB, Faulkin $L$, Jr, Bern HA, Blair PB: Development of mammary tumors from hyperplastic alveolar nodules transplanted into gland-free mammary fat pads of female C3H mice. Cancer Res 1959, 19:515-520.

2. Shackleton M, Vaillant F, Simpson KJ, Stingl J, Smyth GK, Asselin-Labat ML, Wu L, Lindeman GJ, Visvader JE: Generation of a functional mammary gland from a single stem cell. Nature 2006, 439:84-88.
3. Sleeman KE, Kendrick H, Ashworth A, Isacke CM, Smalley MJ: CD24 staining of mouse mammary gland cells defines luminal epithelial, myoepithelial/ basal and non-epithelial cells. Breast Cancer Res 2006, 8:R7.

4. Stingl J, Eirew P, Ricketson I, Shackleton M, Vaillant F, Choi D, Li HI, Eaves CJ: Purification and unique properties of mammary epithelial stem cells. Nature 2006, 439:993-997.

5. Regan JL, Kendrick H, Magnay FA, Vafaizadeh V, Groner B, Smalley MJ: c-Kit is required for growth and survival of the cells of origin of Brca1-mutationassociated breast cancer. Oncogene 2012, 31:869-883.

6. Van Keymeulen A, Rocha AS, Ousset M, Beck B, Bouvencourt G, Rock J, Sharma N, Dekoninck S, Blanpain C: Distinct stem cells contribute to mammary gland development and maintenance. Nature 2011, 479:189-193.

7. Boulanger CA, Wagner KU, Smith GH: Parity-induced mouse mammary epithelial cells are pluripotent, self-renewing and sensitive to TGF- $\beta 1$ expression. Oncogene 2005, 24:552-560.

8. van Amerongen R, Bowman AN, Nusse R: Developmental stage and time dictate the fate of $\mathrm{Wnt} / \mathrm{\beta}$-catenin-responsive stem cells in the mammary gland. Cell Stem Cell 2012, 11:387-400.

9. de Visser KE, Ciampricotti M, Michalak EM, Tan DW, Speksnijder EN, Hau CS, Clevers H, Barker N, Jonkers J: Developmental stage-specific contribution of LGR5(+) cells to basal and luminal epithelial lineages in the postnatal mammary gland. J Pathol 2012, 228:300-309.

10. Williams JM, Daniel CW: Mammary ductal elongation: differentiation of myoepithelium and basal lamina during branching morphogenesis. Dev Biol 1983, 97:274-290.

11. Srinivasan K, Strickland P, Valdes A, Shin GC, Hinck L: Netrin-1/neogenin interaction stabilizes multipotent progenitor cap cells during mammary gland morphogenesis. Dev Cell 2003, 4:371-382.

12. Daniel CW, Silberstein GB: Postnatal development of the rodent mammary gland. In The Mammary Gland: Development, Regulation and Function. Edited by Neville MC, Daniel CW. New York: Plenum; 1987:3-36.

doi:10.1186/bcr3351

Cite this article as: Ordonez L, Smalley MJ: Who do they think they are? Wnt-responsive cells reveal their family trees. Breast Cancer Research 2012 $14: 327$ 